\title{
Zur Strafbarkeit des »goldenen Handschlags« wegen Untreue (§ 266 StGB)
}

- Rechtliche Überlegungen zum Fall Mannesmann -

Die moralische Entrüstung über »goldene Handschläge« für deutsche Manager hat ein Aktenzeichen bekommen. Aus einer der größten Übernahmeschlachten der Wirtschaftsgeschichte wird eine strafrechtliche Fallakte. Keiner der Prozessbeteiligten ist zu beneiden. Die Entscheidung des Falls ist nämlich nicht nur mit kühler juristischer Professionalität - Beweisaufnahme, Beweiswürdigung, schließlich Subsumtion eines festgestellten Sachverhalts unter die Merkmale eines Strafgesetzes - zu bewältigen. Die Prozessbeteiligen haben mediale, wirtschafts- und gesellschaftspolitische Schwerstarbeit zu leisten. Auf ihnen lasten hohe gesellschaftliche Erwartungen, die sich zwischen einem Bedürfnis nach Strafe für scheinbares Fehlverhalten Mächtiger und der ebenso diffusen Angst um den Wirtschaftsstandort bewegen. Es fällt schwer zu glauben, dass die Entscheidungen des Falls, wie immer sie ausfallen mögen, von diesen gesellschaftlichen Erwartungen völlig frei sind. So ist der Fall Mannesmann nicht einmal primär ein strafjuristisches Problem, sondern ein mediales Ereignis. Verfahrenseinstellung, Freispruch, vielleicht auch Verurteilung oder Strafmaß können davon abhängen, wie sich die Prozessbeteiligten kommunikativ präsentieren. Wenn man etwas Besonderes an dem Düsseldorfer Verfahren ausmachen will, dann sind es die verschiedenen Interessen, die sich an das Strafrecht in seinem Zugriff auf deutsche Topmanager richten. Wenn man will, lassen sich ein Interesse an der Funktion des Strafrechts überhaupt (I), natürlich das Entscheidungsinteresse - Strafbarkeit des Verhaltens wegen Untreue oder Freispruch (II) - schließlich das politische Interesse an den Folgen der Entscheidung ausmachen (III).

\section{Modernes Strafrecht - ein Kommunikationsmedium}

Das Strafrecht, behaupten Kriminologen, sei ein Medium der Kommunikation. ${ }^{1}$ In dieser Aussage liegt eine wichtige Beschreibung dessen, wie und warum modernes Strafrecht - scheinbar - funktioniert. Es soll Werte vermitteln, ethische Handlungsorientierung bieten. Strafsanktion und Strafverfahren sollen präventiv wirken, also potentielle Täter abschrecken, Exempel statuieren, den Täter bessern oder die Gesellschaft vor dem Täter sichern. Vor allem aber nutzt die Politik das Strafrecht, um eine durch den Bürger längst verlorengeglaubte Fähigkeit zur Lösung gesellschaftlicher Probleme doch noch zu belegen. ${ }^{2}$ Kaum eine gesellschaftspolitische Debatte vergeht, an deren Ende nicht der Griff zum Strafrecht steht. ${ }^{3}$ Dahinter verbirgt sich ein Muster:

1 Vgl. dazu P.-A. Albrecht, Kriminologie, 2. Auflage, S. 86.

2 AaO., S. 79.

3 Vgl. Voß, Strafe muß nicht sein, in: H. Peters (Hrsg.), Muß Strafe sein?, 1993, 135 ff. (S. 139). 
Wenn man Gesellschaftsprobleme als »Kriminalität«, für die das Strafrecht zuständig ist, deutet, kann man es sich leicht machen. Mit der strafrechtlichen Musterlösung bekommen die Probleme ein Gesicht - das des Angeklagten - und zugleich lassen sich die eigentlichen und mitunter komplizierten Ursachen des Problems hinter dem Strafrecht geschickt verbergen. ${ }^{4}$ Läßt sich das Problem nach Schwarz-Weiß-Kategorien ordnen, kann man die Angeklagten nach Sympathiewerten kategorisieren, kommen politische Strukturen als potentielle Problemverursacher gar nicht mehr zur Sprache. Mit Hilfe der Massenmedien wird dieser problemverstellende Effekt des Strafrechts noch verstärkt. Massenmedien suchen nach Markt und das Strafrecht ist ein gutes Produkt, das sich zum medialen Verkauf eignet. ${ }^{5}$ Die strafrechtliche Fallakte ist im öffentlichen Bild danach spektakulär, dramatisch und Symbol für den Verfall von Sitten und Gebräuchen. Strafrecht und öffentliches Drama gehören zusammen - und dies schadet dem Strafrecht, das auf Behutsamkeit, Distanz, Berechenbarkeit hin angelegt ist, soll es seinen demokratisch-rechtsstaatlichen Grundlagen noch genügen.

So betrachtet ist der Mannesmann-Prozess zunächst einmal ein sehr guter Beleg für modernes Strafrecht: Die öffentliche Hauptverhandlung als Medienereignis klärt über Prozessbeteiligte und Rechtsprobleme kaum auf, sondern verengt in Wahrheit den Blick, verschleiert das Wesentliche. Die Botschaft, die vermittelt wird, ist trivial: Sie lautet, dass man auch die Großen hängen kann, wenn man nur politisch will. Und das Strafrecht erbringt einen Aktivitätsnachweis, den die Politik eher nicht in der Lage ist zu erbringen, nämlich Grenzen zu ziehen für das grenzüberschreitende Handeln von Großkonzernen, einen wirksamen Regelrahmen für Risiken der Globalisierung zu setzen, schließlich auch wirksame Haftungsregeln für Unternehmensvorstände zu begründen. Im Medienlicht des Düsseldorfer Verfahrens wirken die Angeklagten als personifizierbare Verantwortliche für die Ungerechtigkeiten, die mit dem Prozess der Globalisierung verbunden sind. Mit den Managern im strafrechtlichen Zugriff scheinen auch alle Risiken der regelentfesselten Weltwirtschaft unter Kontrolle. Weniger der Inhalt als die Form der Aussage und des Verhaltens der Angeklagten stehen im Fokus des Interesses. Gesten, seien sie auch aus dem Zusammenhang gerissen, können eine kraftvolle Symbolik entfalten, die für sich spricht. Diese Symbolik wird in der öffentlichen Debatte begierig aufgegriffen und verwertet, auch um des parteipolitischen Nutzens willen.

Und der Schaden für das Strafrecht? Er ergibt sich vielleicht erst auf den zweiten Blick. Mit dem strafrechtlichen Vorwurf der Untreue (§266 StGB) lässt sich eine symbolische Wirtschafts- und Finanzpolitik betreiben, die viel verspricht und nichts einlöst.

4 P.-A. Albrecht, aaO., S. 80.

5 AaO., S. 87. 


\section{Strafbarkeitsvoraussetzungen}

Wegen Untreue ( $\$ 266 \mathrm{StGB}$ ) macht sich strafbar, »wer die ihm durch Gesetz, behördlichen Auftrag oder Rechtsgeschäft eingeräumte Befugnis, über fremdes Vermögen zu verfügen oder einen anderen zu verpflichten, missbraucht (...) und dadurch dem, dessen Vermögen er zu betreuen hat, Nachteil zufügt. «Wegen Untreue macht sich auch strafbar, wer »die ihm kraft Gesetzes, behördlichen Auftrags Rechtsgeschäfts oder eines Treueverhältnisses obliegende Pflicht, fremde Vermögensinteressen wahrzunehmen, verletzt und dadurch dem, dessen Vermögen er zu betreuen hat, Nachteil zufügt«. Für normale Bürger - Nichtjuristen - erschließt sich dieser Text nicht, schon gar nicht vermittelt er in verständlicher Weise, welches Verhalten denn der Straftatbestand der Untreue genau im Blick hat, was er verbietet, was er gerade noch erlaubt. Genau genommen existieren zwei Straftatbestände der Untreue: der eine stellt den Missbrauch einer Befugnis, über fremdes Vermögen zu verfügen unter Strafe, wenn daraus für dieses Vermögen ein Nachteil erwächst (§ 266 Abs. 1, 1. Alternative StGB). Der andere bestraft die Verletzung einer Pflicht, die im Hinblick auf das Vermögen eines anderen begründet wurde. Auch hier setzt die Strafbarkeit voraus, dass mit der Pflichtverletzung ein Nachteil für das Vermögen eines anderen verbunden ist (\$266 Abs. 1, 2. Alternative StGB). Gemeinsame Voraussetzung beider Alternativen ist eine bestehende Pflicht, das Vermögen eines anderen zu betreuen. ${ }^{6}$ Strafbar macht sich, wer diese Pflicht verletzt. § 266 StGB erfordert also die Begründung und Verletzung einer Vermögensbetreuungspflicht.

\section{Pflichtenbegründung: Verpflichtungs- und Verfügungsbefugnis}

Eine Vermögensbetreuungspflicht kann sich aufgrund eines Gesetzes, behördlichen Auftrags oder aus Rechtsgeschäft ergeben. Zunächst fällt es im Hinblick auf den Vorstand einer Aktiengesellschaft nicht schwer, die sogenannte $\gg$ Verfügungs- oder Verpflichtungsbefugnis« zu bejahen. Unter Verfügungs- und Verpflichtungsbefugnis versteht man eine Rechtsstellung, die den Täter nach außen in den Stand setzt, Vermögensrechte eines anderen wirksam zu ändern, zu übertragen, aufzuheben oder ihn mit Verbindlichkeiten zu belasten. ${ }^{7}$

$\S \S 77$ Abs. 1 Satz 1, 78 Abs. 1 AktG enthalten die Befugnis des Vorstands zur Geschäftsführung sowie dessen gerichtliche und außergerichtliche Vertretungsmacht. Was der Vorstand an rechtsgeschäftlichen Handlungen unternimmt, wirkt sich unmittelbar auf das Vermögen der Gesellschaft, die er vertritt, aus und zwar unabhängig davon, ob das Mitglied eines Vorstandes alleine oder in Zusammenwirken mit anderen

6 Seit BGHSt 24, 386 ff. gilt die Vermögensbetreuungspflicht nicht nur als Voraussetzung des Treuebruchtatbestands, sondern auch des Missbrauchstatbestands. Dies lässt sich mit der Notwendigkeit begründen, den konturlosen und weiten Anwendungsbereich des Straftatbestands einzuschränken. Die sogenannte Selbständigkeitstheorie dürfte seit einiger Zeit auf dem Rückzug begriffen sein. Vgl. dazu zum Beispiel Nelles, Untreue zum Nachteil von Gesellschaften, 1991, 2. Teil, 3. Kapitel.

7 Schönke/Schröder-Lenckner, 26. Auflage, § 266, Rz. 4. 
handelt. ${ }^{8}$ Ein Vorstandsvorsitzender hat die Befugnis, über das Vermögen der Gesellschaft zu verfügen. Daraus erwächst nicht irgendeine - nebensächliche - Pflicht. Vielmehr zählt die Geschäftsführung zu den Hauptpflichten des Vorstandsmitglieds, die er in eigener Verantwortung ausübt ( $\$ 76 \mathrm{Abs.} 1 \mathrm{AktG})$. Sie stellt daher eine Vermögensbetreuungspflicht gegenüber der Gesellschaft und ihren Mitgliedern im Sinne von $\S 266 \mathrm{StGB}$ dar.

Im Hinblick auf das Mitglied eines Aufsichtsrats bestimmen $\S \S 111$ Abs. 1, 112 AktG, dass der Aufsichtsrat die Geschäftsführung des Vorstandes überwacht (§ 111 Abs. 1 AktG) und die Gesellschaft gegenüber den Vorstandsmitgliedern gerichtlich und außergerichtlich vertritt ( $§ 112$ AktG). Gemäß $§ 111$ Abs. 4 Satz 2 AktG können für eine bestimmte Art von Rechtsgeschäften Zustimmungsvorbehalte des Aufsichtsrats angeordnet werden. Die fehlende Zustimmung hat jedoch - im Unterschied zu $\S \S 183,184$ BGB - nicht die Unwirksamkeit des Geschäfts nach außen hin zur Folge, da die Gesellschaft auch durch einen eigenmächtig handelnden Vorstand berechtigt und verpflichtet wird ( $\$ 78$ Abs. 1, 82 Abs. 1 AktG). § 111 Abs. 4 Satz 2 AktG begründet also kein Handeln mit Außenwirkung. ${ }^{9}$ Folglich besteht auch keine Verpflichtungs- und Verfügungsbefugnis der Mitglieder des Aufsichtsrats im Sinne von $\S 266$ StGB.

Nach $§ 112$ AktG kommt dem Aufsichtsrat aber für alle Rechtsgeschäfte mit Vorstandsmitgliedern eine ausschließliche Vertretungsmacht $\mathrm{zu}^{10}{ }^{10}$ Ein Verstoß gegen $\S 112$ AktG macht das Rechtsgeschäft nichtig, mindestens aber schwebend unwirksam. ${ }^{11}$ Entscheidet der Aufsichtsrat also über die Bezüge der Vorstandsmitglieder, zu denen neben dem Gehalt unter anderem auch Gewinnbeteiligungen, Aufwandsentschädigungen und Leistungen verwandter Art gehören (Art. 87 Abs. 1 AktG), unternimmt er rechtsgeschäftliche Handlungen, welche die Gesellschaft und ihre Mitglieder im Außenverhältnis wirksam verpflichten können. Im Hinblick auf Rechtsgeschäfte mit Vorstandsmitgliedern haben die Mitglieder des Aufsichtsrats damit eine Verfügungs- und Verpflichtungsbefugnis gegenüber der Gesellschaft und ihren Mitgliedern inne. ${ }^{12}$ Geht es um generelle Rechtsgeschäfte, so wäre jedenfalls aus den Sorgfaltsanforderungen, die im Rahmen ihrer Kontrollfunktion nach $\S \S 111$ Abs. 1, 116 AktG an die Aufsichtsratsmitglieder gestellt werden, eine Vermögenswahrnehmungspflicht im Sinne des Treubruchtatbestandes ( $\$ 266$ Abs. 1, 2. Alt. StGB) begründet.

Im Fall Mannesmann hat die Entscheidung des Aufsichtsrats, einzelnen Vorstandsmitgliedern Anerkennungsprämien bzw. Pensionsabfindungen zu gewähren, ein Präsidium getroffen. Gemäß $§ 107$ Abs. 3 Satz 1 AktG kann der Aufsichtsrat seine Aufgaben und Befugnisse Ausschüssen übertragen. Bei der Mannesmann-AG bestand ein ständiger Aufsichtsratsausschuss für Vorstandsangelegenheiten (Präsidium), der

8 Vgl. dazu BGHSt 47, 187 ff. (S. 192).

9 Vgl. auch Hüffer, Aktiengesetz, 5. Auflage, § 111, Rz. 19.

10 Hüffer, aaO., $\S 112$, Rz. 3

11 Hüffer, aaO., § 112, Rz. 7.

12 Vgl. dazu bereits auch Tiedemann, Untreue bei Interessenkonflikten. Am Beispiel der Tätigkeit von Aufsichtsratsmitgliedern, in: Festschrift für Tröndle, 1989, S. 319 ff. 
über die Anstellungsverträge zu entscheiden hatte sowie die Entscheidung über die Gewährung von Krediten an Vorstandsmitglieder treffen konnte. ${ }^{13}$ Damit wird die für den Aufsichtsrat bei Geschäften mit Vorstandsmitgliedern bestehende Verfügungsund Verpflichtungsbefugnis auch auf das Präsidium verlagert. Entsprechend gilt die Vermögensbetreuungspflicht gerade auch für die Mitglieder des Präsidiums.

\section{Pflichtenverletzung: Missbrauch der Verfügungs- und Verpflichtungsbefugnis (§266 Abs. 1, 1. Alt. StGB)}

Entscheidende Voraussetzung des Untreuestraftatbestandes ist die Verletzung einer Pflicht gegenüber dem betreuten Vermögen. § 266 Abs. 1, 1. Alt StGB setzt insoweit den Missbrauch der begründeten Verpflichtungs- und Verfügungsbefugnis voraus. Die Formel, mit der man den Missbrauch definiert, erweist sich als erklärungsbedürftig. Missbrauch nach $§ 266$ Abs. 1, 1. Alt. StGB liegt vor, wenn der Täter im Rahmen seines rechtlichen Könnens die im Innenverhältnis gezogenen Grenzen seines rechtlichen Dürfens überschreitet. ${ }^{14}$ Dem Missbrauch einer Verfügungs- und Verpflichtungsbefugnis liegt damit ein rechtsgeschäftliches Handeln zugrunde, das auch rechtswirksam ist. Handlungen rein tatsächlicher Art unterfallen nicht dem Missbrauchstatbestand. ${ }^{15}$ Entscheidend ist damit zunächst, ob durch die Zahlungen der Anerkennungsprämien bzw. der Pensionsabfindungen das Vermögen der Mannesmann-AG nach außen wirksam und verbindlich verpflichtet wurde. Dann ist entscheidend, ob dieses rechtsgeschäftliche Handeln mit den Verpflichtungen des Vorstandes bzw. des Aufsichtsrates gegenüber der Gesellschaft und ihren Aktionären vereinbar war.

An dieser Stelle drängen die aktien- und gesellschaftsrechtlichen Besonderheiten des Falls in die strafrechtliche Würdigung des Sachverhalts kraftvoll hinein. Zunächst muss man das, was in der Alltagssprache der Medien »goldener Handschlag«, »erhöhte Zuwendung « oder auch untechnisch »Abfindung « heißt, sorgfältig trennen, um die strafrechtlichen Folgen für eine Strafbarkeit nach § 266 StGB differenziert beurteilen zu können: Es geht um »Anerkennungsprämien« (»appreciation awards«) zum einen und »Pensionsabfindungen« zum anderen.

\section{a) Anerkennungsprämien}

Anerkennungsprämien sind Zahlungen, die etwa dann gewährt werden, wenn die Steigerung des Unternehmenswerts honoriert werden soll. Im Fall des Vorstandsvorsitzenden der Mannesmann-AG wird die Anerkennungsprämie dann auch mit der »enormen Wertsteigerung « begründet, welche die Mannesmann-Aktien in Zusammenhang mit dem Übernahmekampf erzielen konnten. ${ }^{16}$ Auch der Aufsichtsratsvorsitzende erhielt die Prämie mit der Begründung, einen wichtigen Beitrag zum »wertmäßigen Erfolg der Aktionäre « geleistet zu haben. ${ }^{17}$ Diese Begründungen haben also mit Leistungen der

13 Nr. 12 der Geschäftsordnung des Aufsichtsrats der Mannesmann-AG von 1972, Nr. 15 GO des AR der MAG vom 17.4.2000, entnommen aus Hüffer, Beilage zu BB 2003, Heft 43, S. 2.

14 Vgl. Schönke/Schröder-Lenckner/Perron, Strafgesetzbuch, 26. Auflage, § 266, Rz. 17.

15 AaO., Rz. 18.

16 Vgl. dazu Hüffer, BB 2003, Beilage Nr. 7, S. 4. 
Vergangenheit zu tun. Bei beiden kommt hinzu, dass auch ihre zukünftige Mitarbeit in dem auf Vodafone übergehenden Unternehmensverbund als besonders bedeutsam eingeschätzt wurde. ${ }^{18}$ Die Anerkennungsprämie weist mithin zwei Komponenten auf: eine Belohnungskomponente - für die Leistungen der Vergangenheit - und eine Anreizkomponente - für zukünftige Leistungen, mögen diese auch für einen aus der Unternehmensübernahme hervorgehenden neuen Unternehmensverbund erbracht werden. ${ }^{19}$

\section{b) Pensionsabfindungen}

Bei den Pensionsabfindungen handelt es sich um »vertragliche Zusagen an pensionsberechtigte Vorstandsmitglieder $«{ }^{20}$ Grundsätzlich ist deren Betrag an einem vorher bestimmten Prozentsatz des letzten Gehalts orientiert. Die Besonderheit im Fall Mannesmann besteht indes darin, dass sich deren Höhe nach dem Gesamteinkommen, das sich aus dem Fixgehalt und flexiblen Bonuszahlungen zusammensetzt, der jeweils aktiven Vorstandsmitglieder richten sollte (Alternativpension). Wegen der positiven Entwicklung des Unternehmens in den Jahren vor der Übernahme, wäre dies die für die Vorstandsmitglieder lukrativere Berechnungsgrundlage gewesen; für das Unternehmen selbst bedeuteten sie jedoch eine Belastung, von der man sich im Zuge der Übernahme befreien wollte. Die letztlich gezahlte Summe der Pensionsabfindungen an Mitglieder des Vorstands entsprang schließlich einem Vergleich, durch den die lebenslang bestehenden Ansprüche der Begünstigten auf die Differenz zwischen den beiden Beträgen der Alternativpension einmalig abgegolten werden sollten. ${ }^{21}$ Entscheidend ist: die Zahlung erfolgte aufgrund eines rechtmäßig begründeten Anspruchs der Vorstandsmitglieder gegen die Gesellschaft.

c) Rechtswirksames Handeln nach außen

Stellt man zunächst auf die Pensionsabfindungen ab, wird klar, dass hier ein gegenüber den Vorstandsmitgliedern rechtsgeschäftlich wirksames Handeln des Aufsichtsrates gegeben war. Die Zahlung erfolgte aufgrund eines bestehenden Anspruchs und nach satzungsmäßigem Durchlauf des Beschlussverfahrens. An der Rechtwirksamkeit der Zahlung von Pensionsabfindungen an Vorstandsmitglieder ist daher nicht zu rütteln. Auch die Staatsanwaltschaft geht in ihrer Anklageschrift von der Rechtswirksamkeit der Präsidiumsbeschlüsse aus, da sie insoweit den Missbrauchstatbestand für einschlägig hält. ${ }^{22}$

Hinsichtlich der Anerkennungsprämien handeln Aufsichtsrat und Präsidium im Rahmen des § 112 AktG. Danach ist ein Rechtsgeschäft mit dem Vorstand jedenfalls schwebend unwirksam, wenn der Aufsichtsrat gesetzes- und satzungswidrig gar nicht mit der Wahrnehmung seiner Befugnisse betraut war. Verstöße gegen einzelne Form-

17 AaO., S. 6.

18 AaO., S. 4 u. S. 6.

19 AaO, S. 3.

$20 \mathrm{AaO}$, S. 6.

$21 \mathrm{AaO}$.

22 Entsprechende Hinweise finden sich bei Hüffer, aaO., S. 34. 
vorschriften des Beschlussverfahrens dürften dagegen das Rechtsgeschäft nicht unwirksam werden lassen und schon gar nicht zu dessen Nichtigkeit führen, wenn dessen Inhalt von den Parteien vernünftigerweise so gewollt war. ${ }^{23}$ Selbst wenn man mit dem Anklagevorwurf tatsächlich solche Formfehler unterstellt, wirkt sich dies nicht auf die Wirksamkeit der Zahlung von Anerkennungsprämien an die Vorstandsmitglieder aus. Damit möglicherweise verbundene Pflichtverletzungen stellen ein Problem des gesellschaftsrechtlichen Innenverhältnisses dar. Auch sie hindern gemäß §§ 112, 116 Satz 1, 78 Abs. 1, 82 Abs. 2 AktG die äußere Wirksamkeit des Rechtsgeschäfts nicht, können im Innenverhältnis aber Schadensersatzansprüche der Gesellschaft und ihrer Mitglieder begründen ( $\S 116$ Satz 1, 93 Abs. 2 Satz 1 AktG). Strafrechtliche Gründe, die eine Nichtigkeit des Rechtsgeschäfts wegen des Verstoßes gegen ein gesetzliches Verbot (zum Beispiel § 299 StGB) auslösen könnten, sind nicht ersichtlich, bzw. wurden im Zwischenverfahren durch das Tatgericht nicht weiter verfolgt. Auch hinsichtlich der Anerkennungsprämien dürfte daher der Missbrauchstatbestand einschlägig sein.

\section{d) Kriterien der Pflichtverletzung}

Die für die Strafbarkeit zentrale Frage lautet dann, ob durch die Zahlung der Anerkennungsprämien oder durch die Pensionsabfindungen eine Verletzung der aus der Verfügungs- und Verpflichtungsbefugnis sich ergebenden Pflichten folgt. Grenzen des innenrechtlichen Dürfens bestimmen sich nach den Sorgfaltspflichten und Verantwortlichkeiten der Aufsichtsratsmitglieder gemäß §§ 116, 93 AktG. Damit ist der Vorwurf der strafrechtlichen Untreue untrennbar mit dem Aktienrecht verbunden.

Die Strafbarkeit wegen Untreue kann sich jedenfalls nur dann ergeben, wenn das Verhalten der Angeklagten aktienrechtswidrig war. Bloße Aktienrechtswidrigkeit alleine kann aber den Straftatbestand der Untreue nicht vollständig begründen. Auch dann, wenn man feststellt, dass das Verhalten der Angeklagten mit den Vorschriften des Aktien- und Gesellschaftsrechts nicht in Einklang steht, müssen weitere Voraussetzungen erfüllt sein, um eine Strafbarkeit wegen Untreue annehmen zu können. ${ }^{24}$ Der Bundesgerichtshof hält in einem neueren Urteil hierzu fest, dass nicht jede gesellschaftsrechtliche Pflichtverletzung auch strafrechtsrelevant ist. ${ }^{25}$ Es muss sich vielmehr um eine gravierende Pflichtverletzung handeln. ${ }^{26} \mathrm{Ob}$ die Pflichtverletzung

23 Dies gilt insbesondere im Hinblick auf die Protokollierung des Beschlusses ( $§ 107$ Abs. 2 Satz 3 AktG) und wohl auch bei Nichtigkeit einer Stimmabgabe, zumindest dann, wenn die Beschlussfassung nicht auf dieser beruht. Vgl. dazu Palandt/Heinrichs, BGB, 63. Auflage, $\S 34$, Rdnr. 2.

24 Die Regelverletzung, auf die das Strafrecht verweist, stellt sich somit als notwendige, aber nicht als hinreichende Bedingung einer Strafbarkeit dar. Das Strafrecht ist insoweit nur limitiert akzessorisch und muss dies auch sein, da nicht jede Regelverletzung eine strafwürdige Rechtsverletzung sein kann. Vgl. zu diesem Problem der Akzessorietät bereits Lüderssen, Ein prokrustes Bett für ungleiche Zwillinge, BB 1996, 2525 ff.; derselbe, FSLampe 2003, 727 ff. (S. 729).

25 BGHSt 47, $187 \mathrm{ff}$.

26 BGHSt 47, 187 ff. (1. Leitsatz). 
gravierend ist, hängt nicht einfach vom Umfang der geleisteten Zahlungen ab. Entscheidend sind weitere gesellschaftsrechtliche Kriterien, wie zum Beispiel die fehlende Nähe der Zahlung zum Unternehmensgegenstand, deren Unangemessenheit im Hinblick auf die Ertrags- und Vermögenslage des Unternehmens, mangelnde Transparenz im Innenverhältnis der Gesellschaft sowie das Vorliegen sachwidriger Motive, insbesondere das Verfolgen rein persönlicher Präferenzen. ${ }^{27}$ Die Strafbarkeit der Aufsichtsrats- und Vorstandsmitglieder hängt also zunächst von zwei Komponenten ab: eine aktienrechtlich begründete Pflichtverletzung und eine strafrechtliche Wertung, dass diese Verletzung »gravierend«, also für das Strafrecht von Relevanz ist.

aa) Aktienrechtswidrigkeit

Eine Pflichtverletzung nach dem Aktienrecht kann sich in formaler und in inhaltlicher Hinsicht ergeben. In formaler Hinsicht ist zu prüfen, ob die Beschlüsse des Präsidiums der Mannesmann-AG ordnungsgemäß zustande gekommen sind. Dies ist auch eine tatsächliche Frage, auf die es an dieser Stelle nicht ankommen soll. Im übrigen gelte hier dann das bei der Frage des nach außen wirksamen rechtsgeschäftlichen Handelns Gesagte: Aus der Verletzung formeller Regeln würde sich eine Aktienrechtswidrigkeit nicht ergeben. ${ }^{28}$ Eine Strafbarkeit nach $\S 266$ StGB kommt dann um so weniger in Betracht als allein die Verletzung formeller Regeln des Beschlussverfahrens grundsätzlich keine gravierende Pflichtverletzung darstellen kann.

Inhaltlich müssen sich die Zahlungen, gerade in ihrer Höhe, auf eine aktienrechtliche Grundlage zurückführen lassen. Dies wird vor allem durch die Staatsanwaltschaft bestritten. Sie trägt vor, dass die Verträge der Vorstandsmitglieder keinen Anspruch auf die Zahlungen enthielten und eine nachträgliche Begründung solcher Ansprüche unzulässig gewesen sei. Darüber hinaus ergebe sich die Pflichtwidrigkeit der Leistungen daraus, dass sie nicht im Unternehmensinteresse gelegen haben. ${ }^{29}$

Das Aktiengesetz lässt eine andere Interpretation zu. Zunächst einmal ist es möglich, dass die bestehenden Anstellungsverträge nachträglich geändert wurden ( $\$ 311$ Abs. 1, 2. Alt. BGB). Eine solche nachträgliche Änderung ist zivilrechtlich nicht zu beanstanden, da keine besonderen aktienrechtlichen Regeln bestehen, die die Möglichkeit von Änderungsverträgen einschränken. ${ }^{30}$ Mangels einer zivilrechtlichen Beschränkbarkeit von Änderungsverträgen, die so ganz dem Grundsatz der Vertragsfreiheit unterliegen, kann eine solche Vertragsänderung auch strafrechtlich nicht als eine Pflichtverletzung angesehen werden. ${ }^{31} \mathrm{Ob}$ der Aufsichtsrat überhaupt einen Vertrag abschließen durfte, ${ }^{32}$ ist keine strafrechtliche, sondern ausschließlich eine zivilrechtliche Frage, ansonsten würde das Strafrecht der Vertragsfreiheit nicht akzeptable Bürden auferlegen. Für die Pensionsabfindungen gilt sogar, dass die Vorstandsmitglieder

27 BGHSt 47, $187 \mathrm{ff}$. (2. Leitsatz).

28 Vgl. oben.

29 Zit. nach den Sachverhaltsangaben von Hüffer, BB 2003, Beilage Nr. 7, S. 16.

30 Vgl. dazu Mertens, Kölner Kommentar, § 84 AktG, Rz. 33 u. Rz. 91.

31 So aber wohl Rönnau/Hohn, NStZ 2004, 113 ff. (S. 120).

32 Vgl. aaO. 
einen rechtswirksamen Anspruch gegen die Gesellschaft hatten, der durch die Einmalzahlung abgegolten wurde.

Beanstandet werden kann aber im Hinblick auf die Anerkennungsprämien, ob diese nachträgliche und konkludent erfolgte Vertragsänderung mit dem Unternehmensinteresse wirklich in Einklang stand. Das Unternehmensinteresse beschränkt so die Entscheidungsfreiheit von Vorstand und Aufsichtsrat. Der Begriff des Unternehmensinteresses jedoch ist weit und kann vor dem Hintergrund unternehmerischer Praxis keineswegs nur auf einzelne Aspekte - etwa der künftigen Anreizwirkung einer Anerkennungsprämie - verengt werden. ${ }^{33}$ Was dem Unternehmensinteresse objektiv entspricht, ist schwierig zu ermitteln. Verschiedene Kriterien dürften bei der konkreten Bestimmung dieses Interesses eine gleichgeordnete Rolle spielen. ${ }^{34}$ Neben dem Bestand und der dauerhaften Rentabilität eines Unternehmens sind die Interessen der Aktionäre, der Arbeitnehmer und der Öffentlichkeit im allgemeinen ebenso bedeutsam. All diese Kriterien müssen Vorstand und Aufsichtsrat bei ihren Entscheidungen gegeneinander abwägen. ${ }^{35}$ Auf Inhalt und Ergebnis der Abwägung kommt es dabei weniger an, als vielmehr darauf, dass überhaupt eine Abwägung stattfand. ${ }^{36}$ Wenig spricht dafür, dass die getroffene Entscheidung gänzlich ohne diese Abwägung vorgenommen wurde. Nur dann, wenn die Zahlung der Anerkennungsprämien den genannten Kriterien durchweg zuwiderliefe, ließe sich auch von einem Ermessensfehler bei der Entscheidung des Aufsichtsrates sprechen, der zu einer völligen Unvertretbarkeit der unternehmerischen Entscheidungen führte.

Bliebe noch die Höhe der geleisteten Zahlungen. Von dem Schaudern über das Aus$\mathrm{ma} ß$ der Summe gilt es sich zu befreien, wenn man beurteilen will, ob die Zahlung auch rechtswidrig war. Das Aktiengesetz schreibt vor, dass die Bezüge von Vorstandsmitgliedern in einem angemessenen Verhältnis zu den Aufgaben des Vorstandsmitglieds und zur Lage der Gesellschaft stehen müssen ( $\$ 87$ Abs. 1 AktG). Bei der Beurteilung der »Angemessenheit « gilt, dass Vorstand wie Aufsichtsrat die »Sorgfalt eines ordentlichen und gewissenhaften Geschäftsleiters« anzuwenden haben (§§ 93 Abs. 1, 116 Satz 1 AktG). Dies sind vage Voraussetzungen, die das Aktienrecht kaum konkretisiert. Darin liegt auch der Grund der in der Öffentlichkeit diskutierten Rechtsauffassungen über das, was im Fall Mannesmann angemessen war. Entscheidend ist, dass Vorstand und Aufsichtsrat eines Unternehmens ein sehr weiter Ermessensspielraum eingeräumt ist, selbst darüber zu befinden, was sie in einer konkreten Lage des Unternehmens noch

33 In diese Richtung zielt die Stellungnahme von Lutter/Zöllner, FAZ vom 10. Februar 2004, S. 12.

34 Die Spannbreite dieser Kriterien findet Ausdruck in den Standards einer guten und verantwortungsvollen Unternehmensführung: Dazu zählen Transparenz und Nachvollziehbarkeit unternehmerischen Handelns, »Vertrauen der internationalen und nationalen Anleger, der Kunden, der Mitarbeiter und der Öffentlichkeit in die Leitung und Überwachung deutscher börsennotierter Aktiengesellschaften« (vgl. Deutscher Corporate Governance Kodex, Präambel). Vgl. auch Corporate Governance Kodex, 4.3.3 Satz 1, 5.5.1 Satz 1.

35 Vgl. dazu Henze, BB 2000, 209 ff. (S. 212); Hopt, ZGR 1993, 534 ff. (S. 536); Kölner Kommentar zum Aktiengesetz-Mertens, § $76 \mathrm{AktG}$, Rz. 19 (»praktische Konkordanz von Verantwortungsrichtungen«). Vgl. auch BGHSt 47, 187 ff. (S. 196).

36 Kölner Kommentar-Mertens, § 76 AktG, Rz. 16.; BGHSt, aaO. 
für angemessen halten. Die Frage ist dann nicht, ob nicht niedrigere Beträge angemessener gewesen wären, sondern ob die Leistung des Vorstandsmitgliedes und die Lage der Gesellschaft gänzlich verkannt wurden. ${ }^{37}$ Setzt man die an den Vorstandsvorsitzenden der Mannesmann-AG geleistete Gesamtzahlung von 30 Millionen Euro in Relation zu dem Bilanzgewinn von Mannesmann im Jahre 2000, der rund 5, 7 Milliarden Euro betrug, oder gar in Relation zu dem Gesamtwert des Übernahmegeschäfts von Vodafone Airtouch in Höhe von 178 Milliarden Euro, ergibt dies einen prozentualen Anteil von 0, $52 \%$ bzw. 0, $0017 \%$. Eine solche Relation zeigt, dass die Betragshöhe nicht völlig außerhalb der Unternehmenswirklichkeit oder völlig jenseits sorgfaltsgemäßer Risikokontrolle lag. Die Betragshöhe spiegelt nur die Realität eines Weltmarktgeschehens wider, das sich gerade im Zeitpunkt der Unternehmensübernahme der Mannesmann-AG durch Vodafone Airtouch auf dem Höhepunkt monströser Kapitalverschiebungen und schwindelerregender Gewinnspannen befand. Die Bestimmung eines leistungsgerechten Marktpreises ist angesichts der unbestritten wirksamen Weltmarktmechanismen praktisch kaum justitiabel. Schon gar nicht erscheint es opportun, die Höhe gezahlter Vergütungen nach einem Kriterium der Marktüblichkeit normativ zu korrigieren. ${ }^{38}$ Darin liegt ein wirtschaftspolitisches Problem, das mit Verteilungsgerechtigkeit zu tun hat - aktienrechtswidrig ist das nicht.

bb) Grobe Sachfremdheit als Erfordernis untreuerelevanter gesellschaftsrechtlicher Pflichtverletzung

Unterstellt man diese Aktienrechtswidrigkeit nun aber, etwa weil man die Vertragsgrundlage der Zahlung für nicht gegeben, das Unternehmensinteresse für nicht gewahrt und das Angemessenheitserfordernis für verletzt hält, muss es sich bei der Verletzung der gesellschaftsrechtlichen Pflicht um eine gravierende Pflichtverletzung handeln, wenn diese im Rahmen des Untreuestraftatbestandes relevant sein soll. Dies setzt eine Gesamtschau von Kriterien voraus, die - wenn sie nicht bereits bei dem Problem des Angemessenheitserfordernisses berücksichtigt wurden - nun auf der Ebene der strafjuristischen Würdigung eine Rolle spielen müssen. ${ }^{39}$ Die oben genannten Kriterien (fehlende Nähe zum Unternehmensgegenstand, Unangemessenheit im Hinblick auf die Ertrags- und Vermögenslage, fehlende innerbetriebliche Transparenz sowie das Vorliegen sachwidriger Motive) müssen dann auch alle - kumulativ - erfüllt sein, um von einer gravierenden Pflichtverletzung sprechen zu können. Fasst man diese

37 Vgl. dazu BGHZ 111, 224 (S. 228 f.); BGH NJW 1992, 2894 (S. 2896). Restriktiver dagegen Lutter/Zöllner, FAZ vom 10. Februar 2004, S. 12: Die Vergütung ausscheidender Vorstandsmitglieder könne sich nur auf die geleisteten Dienste erstrecken, diese seien aber durch die zuvor bereits bewilligte Vergütung abgedeckt.

38 Davon gehen wohl Rönnau/Hohn aus. Vgl. NStZ 2004, 113 ff. (S. 118). Dahinter steckt ein Modell von Strafrecht, das als Interventionsinstrument wirtschaftspolitische Entwicklungen steuern kann. Dieses Präventionsmodell des Strafrechts greift indes nicht: weder in transstaatlicher und schon gar nicht in staatlicher Hinsicht. Vgl. dazu bereits P.-A. Albrecht, Prävention als problematische Zielbestimmung im Kriminaljustizsystem, KritV 1986, 55 ff.; derselbe, Das Strafrecht auf dem Weg vom liberalen Rechtsstaat zum sozialen Interventionsstaat, KritV 1988, $182 \mathrm{ff}$.

39 BGHSt 47, 187 ff. (S. 188 2. Leitsatz). 
Kriterien zusammen, wird ein Erfordernis erkennbar, das zu einer gesellschaftsrechtlichen Pflichtverletzung hinzutritt: Diese muss grob sachfremd sein, um den Tatbestand der Untreue zu erfüllen. ${ }^{40}$ Damit wird dem unternehmerischen Entscheidungsspielraum innerhalb des Tatbestandes Rechnung getragen. Der Straftatbestand der Untreue verbietet unternehmerische Entscheidungen nur dann, wenn sie völlig außerhalb von unternehmerischem Zweck und Nutzen erfolgen. ${ }^{41}$ Die Zahlungen an Vorstands- und Aufsichtsratsmitglieder sind hier aber sichtbar mit dem Unternehmensgegenstand verknüpft und vor dem Hintergrund der Ertrags- und Vermögenslage zu rechtfertigen. Unternehmensintern waren sie transparent, der Aufsichtsrat mit der Beschlussfassung hinreichend befasst, vor allem wurden sie auch als Auszahlungen durch den Vorstandsvorsitzenden selbst offengelegt. ${ }^{42}$ Eigennützige Motive stellen sich als nicht nachweisbar dar. ${ }^{43}$ Unterstellt man also - zu Unrecht - eine gesellschaftsrechtliche Pflichtverletzung, so fehlt es dieser am Erfordernis einer groben Sachfremdheit. Damit liegt eine strafrechtlich relevante Verletzung der den Vorstands- und Aufsichtsratsmitgliedern auferlegten Vermögensbetreuungspflichten nicht vor.

\section{Folgenorientierungen}

Bei einem solchen Freispruch wird die öffentliche Enttäuschung nicht gering sein. Allein die Anklage und das Verfahren haben übersteigerte Erwartungen an das Strafrecht geweckt, die es letztlich nicht erfüllen kann. Gewiss: das offensive Prozessverhalten der Angeklagten verliehe dem Freispruch den faden Beigeschmack einer Kapitulation. Es bliebe aber eine wichtige Erkenntnis übrig, die sich auf die Funktion des Strafrechts selbst erstreckt. Das Strafrecht kann nicht erziehen, es ist auch kein Ventil für Ängste und Ohnmachtsgefühle im Angesicht einer kaum kontrollierbaren Weltwirtschaft. Es muss ethische Kriterien, die an das Verhalten der ökonomisch Mächtigen zu stellen sind, gesellschaftspolitischer Debatte überlassen. Schließlich und vor allem: Die Folgen für Sorgfaltspflichtverletzungen von Vorständen und Aufsichtsräten und die Schäden, die aus rücksichtslosem Gewinnstreben entstehen können, müssen einem präzisierten Aktien- und Gesellschaftsrecht überantwortet werden.

40 Ähnlich bereits Tiedemann, der ein »eindeutig unvertretbares Verhalten« für den Straftatbestand der Untreue voraussetzt. Siehe Tiedemann, Untreue bei Interessenkonflikten. Am Beispiel der Tätigkeit von Aufsichtsratsmitgliedern, Festschrift für Tröndle, 1989, S. 319 ff. (S. 328).

41 Der Bundesgerichtshof bejaht dies etwa für den Fall unentgeltlicher Leistungen an Dritte, die nicht mit dem Unternehmensgegenstand zusammenhängen (Sponsoring von Sport und Kultur), insbesondere dann, wenn diese rein persönlichen Vorlieben des Sponsors dienten. Vgl. BGHSt 47, 187 ff. (S. 197 ff.).

42 Ganz anders dagegen die Sachverhaltskonstellation, die der Entscheidung BGHSt 47, 187 ff. zugrunde lag. Hier wurde die Zuwendung verschleiert, die Auszahlung erfolgte verdeckt und nicht auf dem üblichen Weg über die Hauptbuchhaltung. Vgl. BGHSt 47, 187 ff. (S. 199).

43 Entsprechende Vorwürfe der Anklage wurden nicht für das Hauptverfahren zugelassen und scheinen nun auch durch die Zeugenaussage des Direktors von Hutchison Whampoa widerlegt. Vgl. dazu FAZ vom 27. Februar 2004, Seite 15. 
Bei einer Verurteilung zeichnet sich die Zukunft des § 266 StGB unheilvoll ab. Der Straftatbestand geriete zu einer tagespolitischen Allzweckwaffe. Wirtschaftliche und politische Verantwortungsträger stünden im Fokus strafrechtlicher Kontrolle. Jeder Beratervertrag, jede Verschwendung öffentlicher Gelder, jede satzungswidrige Entgegennahme von Parteispenden ist ein potentieller Fall für den Staatsanwalt. Dies beträfe indes nicht nur die vermeintlich Mächtigen: Auch in den öffentlich nicht beachteten, scheinbar geringfügigen Fällen wird jede weitere Expansion des Untreuestraftatbestandes nachhaltige Auswirkungen haben: auf den GmbH-Geschäftsführer, auf den Vorsitzenden eines Kaninchenzüchtervereins, auf den Kassierer des Ortsbezirks irgendeiner politischen Partei. Auch über ihnen schwebte das Damoklesschwert der Strafbarkeit nach $\S 266$ StGB. Die Untreue entfaltete so ein fast ungebremstes Drohpotential, in dem jedes gesellschaftspolitische und demokratisch unverzichtbare Engagement ersticken kann. Darin liegt nicht nur ein Schaden für den Wirtschaftsstandort eine erschreckend verkürzte Sicht - , sondern für die rechtsstaatlich verfasste Zivilgesellschaft insgesamt. Man mag ein paar »Große« hängen, für die »Kleinen« stehen dann aber Galgen in noch größerer Zahl als bisher bereit! 